\title{
Outcomes after implementing restrictive blood transfusion criteria in extremely premature infants
}

\author{
Daniel Knee ${ }^{1} \cdot$ Serena Knoop ${ }^{2} \cdot$ Alan T. Davis $^{3,4} \cdot$ Brenda Rawson $^{2} \cdot$ Anna DiCarlo $^{5} \cdot$ Rosemary Olivero $^{6}$
}

Received: 1 February 2019 / Revised: 11 April 2019 / Accepted: 29 April 2019 / Published online: 20 June 2019

(c) The Author(s), under exclusive licence to Springer Nature America, Inc. 2019

\begin{abstract}
Objective To assess mortality and morbidities in very low birthweight (VLBW) infants before and after changing to a restrictive blood transfusion guideline (RTG).

Study design This is a large retrospective study comparing outcomes of a liberal transfusion guideline (LTG) and RTG in VLBW infants admitted to a large single neonatal intensive care unit. Blood and platelet transfusion details, mortality, and diagnoses of frequently diagnosed morbidities were collected for each infant.

Results Mortality was similar between RTG and LTG groups $(6.8 \%$ vs. 6.3\%, $p=0.755)$. Rates of periventricular leukomalacia (PVL), retinopathy of prematurity (ROP), sepsis and the diagnosis of necrotizing enterocolitis (NEC) within $48 \mathrm{~h}$ of a PRBC transfusion were significantly lower with RTG $(p<0.05)$. Chronic lung disease was similar between groups.

Conclusion RTG are safe compared to LTG, and are associated with lower rates of PVL, ROP, transfusion-associated cases of NEC and sepsis.
\end{abstract}

\section{Introduction}

Anemia is common in premature infants, especially in those born at less than 30 weeks gestation. An inverse relationship exists between gestational age and receipt of packed red blood cell (PRBC) transfusions: the lower the gestational age at birth, the greater the number of PRBC transfusions received [1]. Nearly $85 \%$ of extremely low

Daniel Knee

Daniel.knee@spectrumhealth.org

1 Neonatal Associates, PHC and Helen DeVos Children's Hospital of Spectrum Health Hospital Group, Grand Rapids, MI, USA

2 Helen DeVos Children's Hospital of Spectrum Health Hospital Group, Grand Rapids, MI, USA

3 Spectrum Health Office of Medical Education Scholarly Activity Support, Grand Rapids, MI, USA

4 Department of Surgery, Michigan State University, Grand Rapids, MI, USA

5 Spectrum Health/Michigan State University/Helen DeVos Children's Hospital Pediatric Residency Program, Grand Rapids, MI, USA

6 Department of Pediatrics and Human Development, Helen DeVos Children's Hospital of Spectrum Health and Michigan State College of Human Medicine, Grand Rapids, MI, USA birthweight (ELBW) infants receive at least one PRBC transfusion before discharge from the neonatal intensive care unit (NICU) [2]. While anemia is expected in premature infants, a debated question remains: At what hemoglobin or hematocrit level should providers transfuse an infant? While blood transfusion is necessary in many circumstances, it has been shown to be an independent risk factor for mortality in critically ill children [3]. The majority of outcome data on restricting PRBC transfusions have been reported in adult populations [4, 5]. Recently published retrospective data report that mortality is similar in restrictive versus liberal transfusion criteria for premature infants [6].

It is well-accepted that PRBC transfusions are not without risks [7], but at what threshold do the benefits outweigh the risks? Transfusion criteria continue to be provider- and institution-dependent, but generally transfusion criteria have been transitioning from a "liberal" to a more "restrictive" approach [7]. The impact of minimizing transfusions on the development of both acute and more chronic diagnoses in premature infants has been a growing area of research [6-13]. One randomized controlled trial of outcomes in infants transfused with various transfusion criteria showed an increased rate of severe neurologic diagnoses in infants transfused with more restrictive guidelines [8]; however, this and other morbidities have not 
been corroborated with other large studies. The growing body of literature on the impact of restricting PRBC transfusions on various neonatal morbidities has yielded conflicting results [6-13], which has prevented a clear standardization of transfusion criteria in the NICU. Our NICU transitioned to restrictive transfusion criteria in 2014. The purpose of our study was to assess differences in mortality and morbidities in very low birthweight (VLBW) infants before and after changing to a restrictive blood transfusion guidelines (RTG).

\section{Materials/subjects and methods}

We performed a retrospective study of VLBW infants (birthweight less than $1500 \mathrm{~g}$ ) admitted to the Gerber Foundation Neonatal Center at Helen DeVos Children's Hospital (HDVCH) of Spectrum Health Hospital from January 1, 2012 to December 31, 2015. The 108-bed NICU at HDVCH is the only level 4 NICU in West Michigan and cares for infants born both within and outside of the institution. The Institutional Review Board at Spectrum Health approved this research.

\section{Study population}

Eligible infants were those with birth weights less than $1500 \mathrm{~g}$, and either born at $\mathrm{HDVCH}$ or transported to HDVCH within the first 2 weeks of life. All included infants had to have been transported to $\mathrm{HDVCH}$ prior to receiving a PRBC transfusion. Infants were excluded if they were diagnosed with congenital heart disease, genetic anomalies, major malformations, or if they died within $48 \mathrm{~h}$ of birth. Infants with red blood cell alloimmunization were not excluded. There were $\sim 860$ infants born during the study time period that were potentially eligible for inclusion. If an infant had repeated admissions, only the data from the initial admission were included.

\section{Intervention}

Infants received PRBC transfusions (leukocyte-depleted and CMV-negative) according to our NICU's transfusion guidelines. Whether to suspend or continue enteral feeding during the time of transfusion was at the discretion of the neonatologist; however, the standard was to continue the infant's current feeding plan during and after transfusion. During the study time period, the standard infusion time for a single PRBC transfusion was $3 \mathrm{~h}$. Neonatologists may have transfused infants outside the guidelines depending on clinical indications. The PRBC liberal transfusion guidelines (LTG) were used during 2012-2013 and RTG were used during 2014-2015 (Table 1). The platelet transfusion

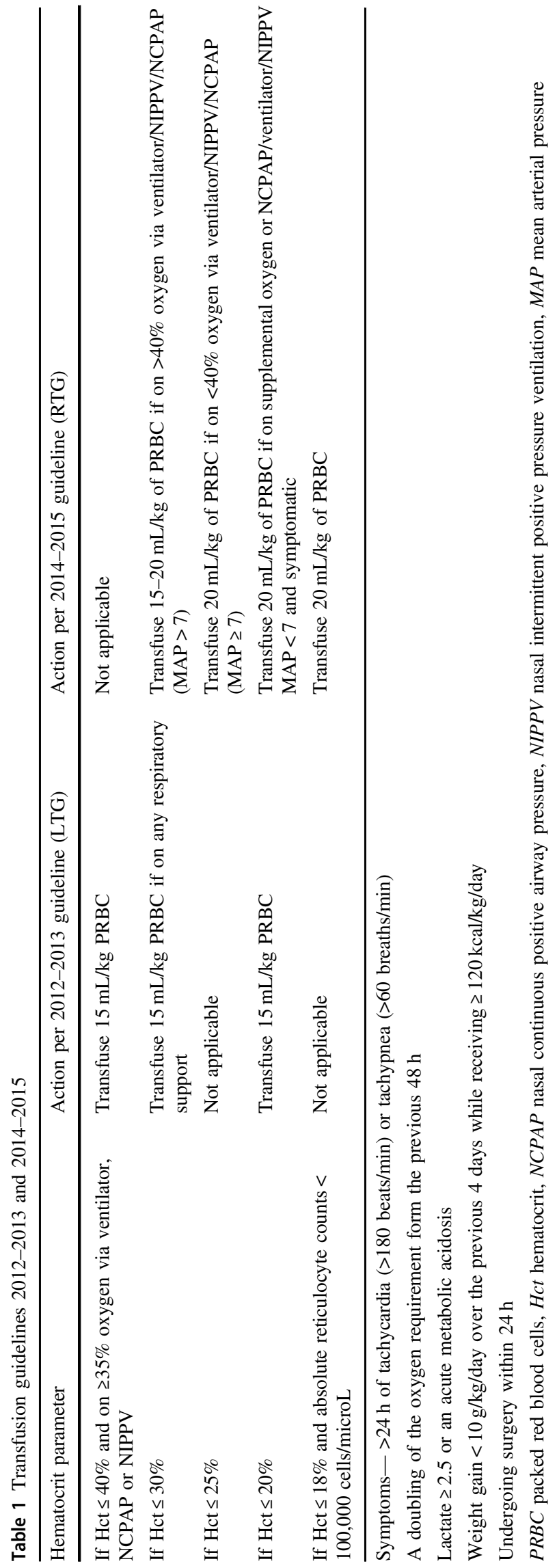




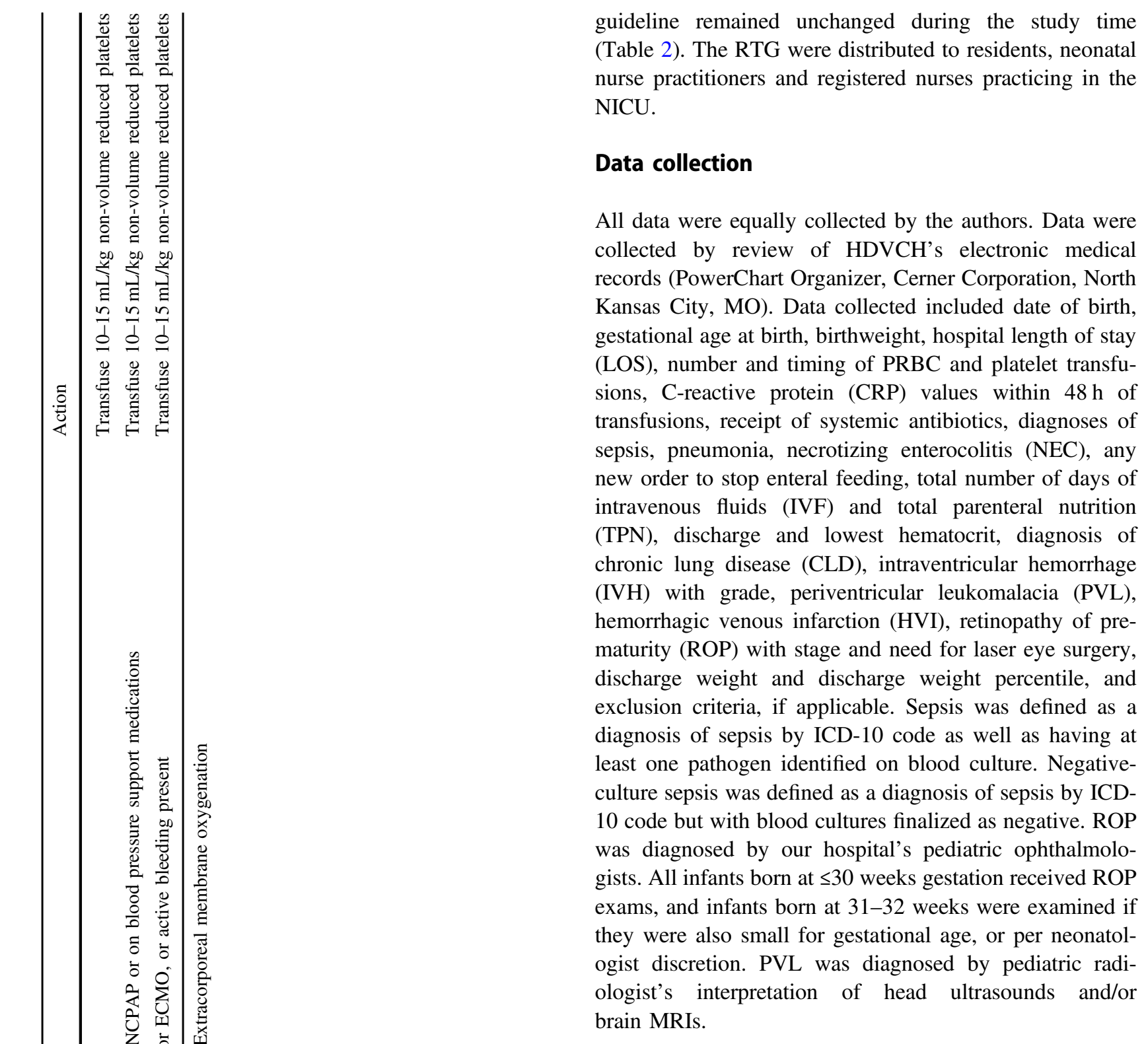

guideline remained unchanged during the study time (Table 2). The RTG were distributed to residents, neonatal nurse practitioners and registered nurses practicing in the NICU.

\section{Data collection}

All data were equally collected by the authors. Data were collected by review of HDVCH's electronic medical records (PowerChart Organizer, Cerner Corporation, North Kansas City, MO). Data collected included date of birth, gestational age at birth, birthweight, hospital length of stay (LOS), number and timing of PRBC and platelet transfusions, C-reactive protein (CRP) values within $48 \mathrm{~h}$ of transfusions, receipt of systemic antibiotics, diagnoses of sepsis, pneumonia, necrotizing enterocolitis (NEC), any new order to stop enteral feeding, total number of days of intravenous fluids (IVF) and total parenteral nutrition (TPN), discharge and lowest hematocrit, diagnosis of chronic lung disease (CLD), intraventricular hemorrhage diagnosis of sepsis by ICD-10 code as well as having at least one pathogen identified on blood culture. NegativeICD almoloric radibrain MRIs.

\section{Statistical methods}

This study was designed following a two-proportion noninferiority or superiority test design. The primary outcome variable for this study was mortality. Summary statistics were calculated. Quantitative, normally distributed data are expressed as the mean $\pm \mathrm{SD}$, while non-normally distributed data are expressed as the median, with the range (25-75th percentile) in brackets. Nominal data are expressed as a percentage. Comparisons between groups for quantitative variables were performed using the $t$-test, with the exception of LOS, which was analyzed using negative binomial regression. With regards to the $t$-test, those comparisons were performed using the two-tailed $t$ test for equal variances, with the exception of the analysis comparing the transformed data for the PRBC, which was 
performed using the two-tailed $t$-test for unequal variances. Prior to analysis, non-normally distributed quantitative data were either log-transformed or transformed using the inverse hyperbolic sine function. Nominal variables were evaluated using the Chi-square test. We separately compared rates of IVH, HVI, PVL, ROP, CLD, sepsis, pneumonia and NEC in the LTG and RTG groups of infants who never received a transfusion. Significance was assessed at $p<0.05$. Data were analyzed using Stata v.15.1 (StataCorp, College Station, TX).

\section{Primary outcome}

The primary outcome was to compare all-cause mortality in RTG versus LTG in VLBW infants.

\section{Secondary outcomes}

Secondary outcomes were to compare discharge weight and percentile, days of TPN and IVF, as well as rates of sepsis, NEC, IVH, ROP and CLD in VLBW infants treated with RTG versus infants treated with LTG.

\section{Results}

\section{Study population}

A total of 865 infants met the gestational age, birthweight and birth date criteria. Of these, 99 infants were excluded from data analysis. Of the 766 patients included in the study, a total of 384 were in the LTG group, and a total of 382 were in the RTG group. Both groups were similar in gestational age, sex and birthweight (Table 3). There was no significant difference in median hospital LOS between the RTG and LTG groups, which was 62.5 days for both groups (data not shown).

\section{Transfusion}

The numbers of PRBC transfusions were significantly different in the two groups (Table 4). The overall number of infants transfused in the LTG group was $60.2 \%$, whereas $39.8 \%$ of the infants in the RTG group received at least one PRBC transfusion $(p<0.001)$. The difference in transfusion rates between RTG and LTG groups was fairly consistent across infants of various gestational ages (Fig. 1). The median number of PRBC transfusions received per infant was also significantly less in the RTG group compared to the LTG group $(2[1-5]$ vs. 4 [2-8], $p<0.001)$. Although the RTG group received fewer PRBC transfusions and had lower "lowest hematocrits" measured, the RTG group had similar discharge hematocrit levels compared to the LTG
Table 3 General demographic data

\begin{tabular}{llll}
\hline & LTG & RTG & $P$ value \\
\hline Gestational age & $27.9 \pm 2.9$ & $28.1 \pm 3.3$ & 0.191 \\
Birthweight & $1045 \pm 293$ & $1077 \pm 283$ & 0.132 \\
Sex, \% female & $(173 / 384) 45.0 \%$ & $(181 / 382) 47.4 \%$ & 0.491 \\
\hline
\end{tabular}

$L T G$ liberal transfusion guidelines, $R T G$ restrictive transfusion guidelines

Table 4 Hematologic data ${ }^{a}$

\begin{tabular}{llll}
\hline & LTG & RTG & $P$ value \\
\hline Lowest hematocrit (\%) & $27.4 \pm$ & $25.8 \pm 6.3$ & 0.012 \\
& 11.0 & & \\
Discharge hematocrit (\%) & $31.0 \pm 6.0$ & $31.6 \pm 5.5$ & 0.143 \\
PRBC transfusions per patient $^{\mathrm{b}}$ & $2(0-5)$ & $0(0-2)$ & $<0.001$ \\
Platelet transfusions per patient $^{\mathrm{b}}$ & $0(0-0)$ & $0(0-0)$ & 0.923 \\
PRBC transfusions per patient if $_{\text {transfused PRBC }}$ & $4(2-8)$ & $2(1-5)$ & $<0.001$ \\
\hline
\end{tabular}

$L T G$ liberal transfusion guidelines, $R T G$ restrictive transfusion guidelines, $P R B C$ packed red blood cells

${ }^{\mathrm{a}}$ Quantitative data expressed as either the mean $\pm \mathrm{SD}$, or as the median (25-75th percentile)

${ }^{\mathrm{b}}$ All patients in the LTG and RTG groups regardless of transfusion history

${ }^{\mathrm{c}}$ Patients who received at least one transfusion

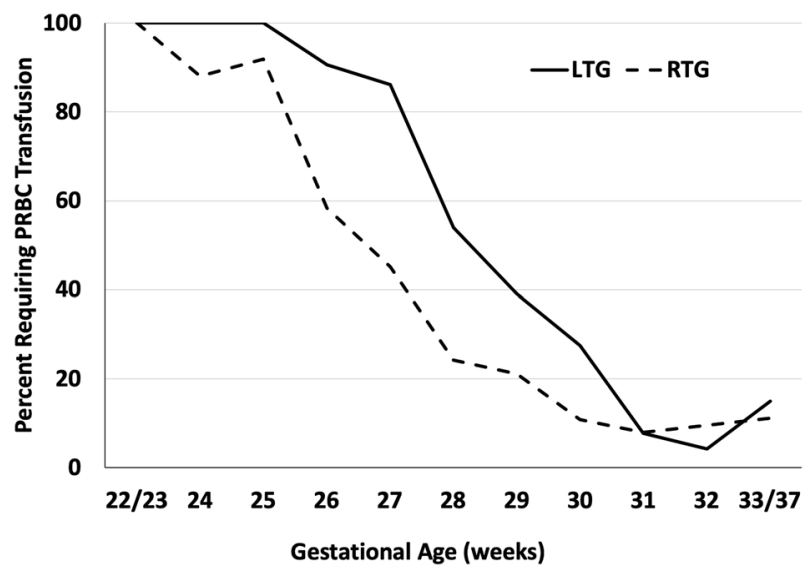

Fig. 1 Differences in PRBC transfusion rate between the LTG and RTG groups by gestational age. PRBC packed red blood cells, LTG liberal blood transfusion guidelines, RTG restrictive blood transfusion guidelines

group. The numbers of platelet transfusions were similar in the LTG and RTG groups.

\section{Primary outcome}

Our primary outcome, all-cause mortality, was similar between LTG and RTG groups (Table 5). The highest 
Table 5 All-cause mortality

\begin{tabular}{lcccc}
\hline GA & LTG & RTG & RR $(95 \%$ CI $)$ & $P$ value \\
\hline $22-25$ weeks & $17 / 101(16.8 \%)$ & $15 / 81(18.5 \%)$ & $1.10(0.59-2.07)$ & 0.766 \\
$26-28$ weeks & $4 / 125(3.2 \%)$ & $4 / 120(3.3 \%)$ & $1.04(0.27-4.07)$ & 0.953 \\
$>28$ weeks & $3 / 156(1.9 \%)$ & $7 / 180(3.9 \%)$ & $2.02(0.53-7.69)$ & 0.290 \\
All Subjects & $24 / 384(6.3 \%)$ & $26 / 382(6.8 \%)$ & $1.09(0.64-1.86)$ & 0.755 \\
\hline
\end{tabular}

$G A$ gestational age, $L T G$ liberal transfusion guidelines, $R T G$ restrictive transfusion guidelines, $R R$ Relative risk, $C I$ Confidence interval

mortality was observed in both groups in infants with the lowest gestational ages at birth (22-25 weeks gestation). When the mortality of all VLBW infants were pooled, the LTG group had all-cause mortality of $6.3 \%$, which was similar to $6.8 \%$ in the RTG.

\section{Secondary outcomes}

Discharge weight and percentile were similar between the LTG and RTG groups (data not shown). The number of days that infants received IVF or TPN was significantly less with RTG compared to LTG (12 days [7-23] vs. 18 days [10-23], $p<0.001)$. The diagnosis of sepsis after 3 days of life was significantly lower with RTG compared to LTG $(11.5 \%$ [44/382] vs. $18.0 \%$ [69/384], $p=0.012$ ) (Table 6). For an episode of sepsis at anytime the prevalence of negativeculture sepsis was $57.1 \%$ (124/217), with the same proportions of negative-culture sepsis in the RTG and LTG groups (57.1\% [44/77] vs. 57.1\% [80/140]). Pneumonia at anytime or within $48 \mathrm{~h}$ of receiving $\mathrm{PRBC}$ was not significantly different between the two groups. The diagnosis of NEC at anytime trended lower with RTG but the difference did not reach statistical significance. NEC diagnosis within $48 \mathrm{~h}$ of a PRBC transfusion was significantly lower in the RTG group compared to the LTG group ( $2.4 \%$ vs. $5.2 \%, p=0.039)$.

When compared to the LTG group, neurologic morbidities of IVH grade 1, 2 and 3, and HVI all trended lower in the RTG group, but the differences did not reach statistical significance (Table 7). Rates of PVL were significantly lower with RTG compared to LTG $1.3 \%$ [5/382] vs. $6.3 \%$ [24/384], $p<0.001)$. The aggregated rates of ROP were significantly lower in the RTG compared to the LTG group (38.0\% [177/384] vs. 46.1\% [145/382], $p=0.015)$. There were no significant differences in rates of either PVL or ROP between the LTG and RTG groups for infants who did not receive a PRBC transfusion (data not shown).

The rates of CLD were similar between RTG and LTG groups. However, CLD rates were significantly higher in infants in the RTG group who did not receive a PRBC transfusion compared to infants in the LTG group who did not received a PRBC transfusion (13.5\% [31/230] vs. 5.9\% [9/153], $p=0.017)$. For infants with CLD, the PRBC transfusion rate was significantly higher in the LTG group,
Table 6 Infectious disease diagnoses data

\begin{tabular}{llll}
\hline & LTG & RTG & $P$ value \\
\hline Sepsis at $>3$ days of life & $69 / 384(18.0 \%)$ & $44 / 382(11.5 \%)$ & 0.012 \\
Pneumonia anytime & $39 / 384(10.2 \%)$ & $30 / 382(7.9 \%)$ & 0.266 \\
$\begin{array}{l}\text { Pneumonia within 48 h } \\
\text { of PRBC }\end{array}$ & $10 / 384(2.6 \%)$ & $9 / 382(2.4 \%)$ & 0.825 \\
NEC anytime & $40 / 384(10.4 \%)$ & $32 / 382(8.4 \%)$ & 0.333 \\
$\begin{array}{l}\text { NEC within 48 h } \\
\text { of PRBC }\end{array}$ & $20 / 384(5.2 \%)$ & $9 / 382(2.4 \%)$ & 0.039 \\
\hline
\end{tabular}

$L T G$ liberal transfusion guidelines, $R T G$ restrictive transfusion guidelines, $P R B C$ packed red blood cells, $N E C$ necrotizing enterocolitis

Table 7 Neurologic outcomes

\begin{tabular}{lccc}
\hline & LTG & RTG & $P$ value \\
\hline IVH & & & \\
0 & $295 / 384(76.8 \%)$ & $314 / 382(82.2 \%)$ & 0.191 \\
1 & $34 / 384(8.9 \%)$ & $20 / 382(5.2 \%)$ & \\
2 & $30 / 384(7.8 \%)$ & $25 / 382(6.5 \%)$ & \\
3 & $25 / 384(6.5 \%)$ & $23 / 382(6.0 \%)$ & \\
HVI & $31 / 384(8.1 \%)$ & $21 / 382(5.5 \%)$ & 0.157 \\
PVL & $24 / 384(6.3 \%)$ & $5 / 382(1.3 \%)$ & $<0.001$ \\
ROP & & & \\
0 & $207 / 384(53.9 \%)$ & $237 / 382(62.0 \%)$ & 0.015 \\
1 & $69 / 384(18.0 \%)$ & $59 / 382(15.5 \%)$ & \\
2 & $58 / 384(15.1 \%)$ & $43 / 382(11.3 \%)$ & \\
3 & $38 / 384(9.9 \%)$ & $41 / 382(10.7 \%)$ & \\
4 & $12 / 384(3.1 \%)$ & $2 / 382(0.5 \%)$ & \\
\hline
\end{tabular}

$L T G$ liberal transfusion guidelines, $R T G$ restrictive transfusion guidelines, IVH intraventricular hemorrhage, HVI Hemorrhagic venous infarction, $P V L$ periventricular leukomalacia, $R O P$ retinopathy of prematurity

compared to the RTG group (93.9\% [138/147] vs. $77.5 \%$ [107/138], $p<0.001)$

\section{Discussion}

As PRBC transfusion is a common occurrence in VLBW infants, it is paramount to determine the optimal threshold 
for transfusing, and whether avoidable transfusions are associated with mortality or comorbidities that often have lifelong consequences. Our primary outcome, all-cause mortality, was not significantly different between the LTG and RTG groups, even when subgroups of gestational ages were compared. Similar findings were reported by Heeger et al. in their retrospective study comparing outcomes in LTG and RTG [6]. In Heeger's study, VLBW infants in the LTG group had a mortality rate of $8.1 \%$ compared to $5.5 \%$ in the RTG group, which was not statistically significant. As survival has dramatically improved in VLBW over the past few decades, it has become evident that mortality, while incredibly important, is perhaps not the most relevant outcome of interest. Our study adds to the growing body of literature examining the impact of tailoring our medical interventions to more precisely improve long-term morbidities in VLBW infants.

Comparing our study results to Heeger et al., both studies demonstrated decreased transfusions in the RTG group [6], as would be expected. Both studies indicate transfusion rates far lower than historic transfusions rates $[6,14]$. Both studies were done in clinical settings that used respiratory support as well as hemoglobin (or hematocrit) levels for transfusion criteria. However, the study site of Heeger et al. used chronological age as a determining factor for transfusion criteria: transfusions were most liberal from birth to one week of life, and were most restrictive in infants greater than two weeks of life [6]. Despite having globally more restrictive transfusion criteria in our study, the percentage of infants transfused was higher in our study (60.2\% compared to $37.5 \%$ in LTG and $39.8 \%$ compared to $32.7 \%$ in RTG). Transfusion rates may have been impacted by the lower mean EGA in our study (28 weeks vs 29 weeks in Heeger et al.), as well as possible differences in iatrogenic blood losses or other clinical phenomena between the two centers. Phlebotomy losses, which significantly impact anemia in VLBW infants with losses as high as $40-80 \mathrm{~mL} / \mathrm{kg}$ ) [15], were not taken into account in either study.

Neurologic and ophthalmologic morbidities including IVH, PVL and ROP have lifelong consequences and may be associated with PRBC transfusion. Studies to date have not conclusively shown differences in rates of most of these phenomena when comparing LTG to RTG practices $[8,11,16]$. Bell et al. observed increased rates of IVH grade 4 combined with PVL in their RTG $[8,16]$. Our rates of HVI and IVH (of all grades) were lower in the RTG group; however, the differences did not achieve statistical significance. IVH is a morbidity that occurs within the first 3 days of life [17]. Our NICU's standard is to obtain a head ultrasound at 7 days of life for infants born with a birthweight of less than $1250 \mathrm{~g}$ unless clinically indicated sooner. While we did not record which day of life PRBC transfusions were given in our study, it is highly likely that infants received PRBC transfusions well after the third day of life, which is when the majority of IVH occurs. Interestingly, the PVL rates in our study were much lower in the RTG group. In contrast to IVH and HVI, PVL is thought to be a consequence of ischemia or inflammation. PVL is not present at birth and is often not detectable early in an infant's hospital course. Infants exposed to repetitive PRBC transfusions are exposed to repetitive episodes of inflammation [18], and we theorize that these repeated inflammatory episodes are a factor causing an increase in PVL.

There are two major ongoing studies (Effects of Transfusion Thresholds on Neurocognitive Outcome of Extremely Low Birth Weight Infants [ETTNO] [19] and the Transfusion of Prematures [TOP] Trial [20]), which may further enlighten the long-term consequences or benefits of using restrictive transfusion criteria. The ETTNO study is a multicenter trial being conducted in Germany comparing death or major neurodevelopmental impairment at 24 months in extremely premature VLBW infants in LTG and RTG groups [19]. The TOP study is comparing cognitive delay, cerebral palsy, severe vision impairment, severe hearing impairment and death at 22-26 months follow up in extremely premature VLBW infants in LTG and RTG [20]. These important trials will further decipher whether restrictive transfusion practices impact long-term neurologic sequelae.

Our ROP rates taken as a whole showed significantly lower ROP rates in our RTG group. Lower incidence of ROP stage 1, 2 and 4 were present in the RTG group, but a slightly higher incidence of stage 3 ROP. Our findings are consistent with a recently published large retrospective study by Lust et al [12]. Lust et al. demonstrated that early PRBC transfusions (at less than 10 days of life) were associated with a four-fold increase in severe ROP, and that an increased number of PRBC transfusions were associated with severe ROP [12]. Our institution's RTG provides the same transfusion criteria regardless of chronological age, thereby decreasing PRBC transfusions throughout the NICU stay (including the first ten days of life). With our RTG criteria our unit observed a 6-fold decrease in stage 4 ROP. Similar to PVL, the cause of ROP is multifactorial, including states of inflammation [21]. Again, with increased transfusions, infants are exposed to an increased number of inflammatory episodes, which may be partially responsible for our higher observed rates of ROP with LTG.

The impact of PRBC transfusion on the development of CLD has also been previously investigated. Rashid et al. reported on the possibility of neonatal post-transfusion lung injury (NPTLI) [13]. In their population of neonates receiving intensive or intermediate care, NPTLI occurred following $8.3 \%$ of transfusions, with an increased $\mathrm{FiO}_{2}$ needs persisting in $7.8 \%$ of all PRBC recipients [13]. This study did not focus specifically on premature infants, which 
is an important limitation. Studies comparing LTG to RTG PRBC transfusion thresholds in VLBW infants have not shown difference in rates of CLD or ventilator days $[8,11,16]$. Our study did not find different CLD rates when comparing LTG and RTG groups. Our PRBC transfusion protocol, like other NICU protocols, includes respiratory support as criteria for transfusion levels $[8,11,16]$. This is in contrast to common adult protocols, which often use only hemoglobin levels as criteria for transfusion [22]. In our study, infants diagnosed with CLD received more PRBC transfusions during their NICU stay compared to infants not diagnosed with CLD, likely because we have more liberal transfusion criteria for an infant on oxygen or ventilator support. Of our infants diagnosed with CLD, 78\% received at least one PRBC in the RTG group and 94\% received at least one PRBC transfusion in the LTG group. Because we use respiratory status as part of our transfusion guideline, infants on oxygen therapy are exposed to additional inflammatory episodes. Therefore, we postulate that the increased rate of transfusions due to using respiratory criteria in our guidelines reduces possible benefits that a restrictive guideline may have on CLD.

Necrotizing enterocolitis continues to be a leading cause of death in extremely premature infants, with extreme prematurity defined as birth between $220 / 7$ weeks to 28 6/ 7 weeks gestation. In 2000-2011, death rates in extremely premature infants due to infectious and pulmonary causes decreased, whereas death rates due to NEC increased [23]. Similar to anemia and the need for transfusion, NEC has an inverse relationship with gestational age [24]. In an effort to reduce NEC rates, our NICU has made several practice changes. Prior to this study, our unit introduced an all breastmilk diet in infants born at less than 32 weeks gestation weighing less than $1500 \mathrm{~g}$ (maternal breastmilk, donor breastmilk and Prolacta), using a standardized feeding grid. For the purposes of our study, NEC was defined by a clinical diagnosis of NEC by a neonatologist and treated for a minimum of 7 days of bowel rest and antibiotics. Our NEC rates of $10.4 \%$ in the LTG and $8.4 \%$ in the RTG were higher than our reported Vermont Oxford Database (VON) average rate between $5 \%$ and $6 \%$. The difference in NEC rates in our study compared to VON data is due to differing radiographic case definition diagnosis criteria used by VON.

NEC is a multifactorial disease process, and it is currently unclear if severe anemia and subsequent tissue ischemia increases the risk for, or even causes NEC, or rather, if PRBC transfusion itself causes NEC. A major challenge to determining causality between anemia and transfusion with NEC is the heterogeneity in diagnosing NEC. Necrotizing enterocolitis is largely a clinical diagnosis, with varying features, clinical spectra and shifting case definitions [24]. Transfusionassociated NEC secondary to tissue reperfusion injury in the setting of significant anemia is not supported by our data, as the RTG group had both lower hematocrit levels and a lower rate of NEC. A recent study by Patel et al. contradicts our findings, concluding that severe anemia is a risk factor for NEC [25]. Patel et al.'s definition of severe anemia was a hemoglobin of $<8 \mathrm{~g} / \mathrm{dL}$. Although infants exposed to RBC transfusions in Patel's study had a higher cumulative rate of NEC than those who were not exposed, PRBC transfusions were not associated with an increased risk of being diagnosed with NEC within the week after PRBC transfusion. Severe anemia, however, was associated with an increased risk for NEC. It is difficult to compare our results to Patel's study, as our transfusion criteria in both the LTG and RTG groups for non-respiratory support was a hematocrit of $18-20 \%$ or approximate hemoglobin of $6.8 \mathrm{~g} / \mathrm{dL}$. Moreover, our study had higher rates of breastmilk exposure and lower rates of antibiotic exposure than in Patel's study; both of these factors are likely to be important in the pathogenesis of NEC.

Regardless of the uncertainty of severe anemia being associated with NEC, transfusion-associated NEC has been well-described [9, 26-28], and may be present in 25-30\% of all NEC cases [26]. While Gephart et al. observed a temporal link between PRBC transfusion and NEC in VLBW infants (with NEC Stage 2a or greater diagnosed within $24-48 \mathrm{~h}$ of transfusion in $15 \%$ of infants studied [26]), meta-analyses of several studies have revealed conflicting results on this association [28]. Our lower rate of NEC with RTG compared to LTG may have been due to the lower number of episodes of NEC associated with a PRBC transfusion. There were 11 fewer PRBC transfusionassociated NEC cases in the RTG group compared to the LTG group. The rate of PRBC transfusion-associated NEC is roughly $2 \%$ per transfusion [26]. In our study there were 1254 PRBC transfusions and 20 cases of NEC within $48 \mathrm{~h}$ of the transfusion in the LTG for a rate of $1.6 \%$ per transfusion. In the RTG, there were 561 PRBC transfusions and nine cases of NEC within $48 \mathrm{~h}$ of transfusion for a rate of $1.6 \%$ per transfusion. As shown in our data, when the association of NEC with transfusions occurs at a fixed rate, then the number of NEC cases associated with PRBC transfusions is directly proportional to the number of transfusions that are given. We theorize that increased exposure to PRBC transfusions may indeed be one of several inciting events in NEC, and that RTG may indeed be preventative. Larger studies with standardized definitions of NEC are needed to further elucidate this possible risk.

Another area of uncertainty is whether PRBC transfusion causes an inflammatory state that can be misdiagnosed and treated as sepsis and/or NEC. Our rate of sepsis at anytime and our rate of negative-culture sepsis were similar between the two groups. However, sepsis occurring after three days of life occurred at a higher rate in the LTG group compared to the RTG group. The treatment of sterile inflammation or 
"negative-culture" sepsis leads to unnecessary microbiological studies, cessation of enteral feeds and use of intravenous antibiotics. Ellefson et al. observed that PRBC transfusions led to a statistically significant increase in absolute blood monocyte and band count especially in infants who were not being enterally fed [18]. Another small study measured the plasma cytokine levels and endothelial activation $2-4 \mathrm{~h}$ after PRBC transfusion and found a statistically significant elevation in IL-1 $\beta$, IL-8, TNF- $\alpha$ and monocyte chemoattractant protein, as well as soluble intracellular adhesion molecule-1 [29]. For the purposes of our study, sepsis was defined as treatment with antibiotics for a minimum of 7 days with or without a positive culture. Immune activation following PRBC transfusion, if clinically apparent, is an important factor to recognize, as sepsis evaluations often result in broadspectrum intravenous antibiotics.

Our findings of increased morbidities with more liberal PRBC transfusion are consistent with recent studies looking at liberal platelet transfusions. A 2005 study found increased morbidities in infants with NEC if their platelet count was maintained at greater than $150,000 / \mathrm{mL}$ rather than $50,000 / \mathrm{mL}$ [30]. Their theory was that platelet transfusions contain bioactive substances, such as the cytokine PAF. These cytokines cause the need for further transfusions, by increasing platelet consumption and inflammatory bowel wall injury [30]. Similar findings were reported in a recent randomized study by Curley et al., which found that liberal platelet transfusion criteria resulted in an increased mortality rate and increased major bleeding episodes when compared to restrictive platelet transfusion criteria [31]. It is plausible that both PRBC and platelet transfusions cause inflammatory processes that warrant further investigation, and further attention to platelet transfusion thresholds is needed.

Cost savings is an additional benefit of restrictive PRBC transfusion protocols. In our study population, the actual number of transfusions dropped from 1254 to 561 over a 2 year window, which equates to a $56 \%$ decrease. A conservative estimated cost per transfusion (hospital cost) is $\$ 210$ per transfusion. Thus, the total savings over the 2 years was $\$ 145,530$. Episodes of sepsis and NEC were also decreased with RTG, which would also add significant cost savings, as these diagnoses are known to significantly add to hospital costs [24].

We acknowledge that our study has several limitations. The primary concern is the retrospective nature of our study. It is possible that during the timeframe of our study, other changes in hospital protocols could have accounted for some of the effects seen. For example, our unit opened a small baby unit (SBU) during the study. The SBU opened 9 months prior to the end of the RTG portion of the study. We statistically evaluated the SBU data separate from the other 15 months of RTG data and found no significant differences; therefore, the RTG data were included as a single 24 month time period. Another change that occurred during the study was that our neonatology staff had two new hires for the second 12 months of the RTG study timeframe. A randomized study would better address the changing of transfusion without timing differences between the groups. Another possible limitation is with reviewing documentation. We relied on the attending neonatologist diagnoses of NEC as well as pneumonia and sepsis (culture negative) without standardized diagnoses criteria. Again, randomized studies with clear diagnosis criteria would better address these secondary outcomes. A final concern is that our results are from a single institution.

RTG is safe in the neonatal VLBW population. RTG when compared to LTG is associated with similar mortality rates and CLD. RTG appears to be protective in regards to ROP, PVL, sepsis and NEC. Further studies to determine long-term neurodevelopment outcomes are ongoing. Studies looking at transfusion guidelines without respiratory support criteria as well as with and without age criteria are also needed.

\section{Compliance with ethical standards}

Conflict of interest The authors declare that they have no conflict of interest.

Publisher's note: Springer Nature remains neutral with regard to jurisdictional claims in published maps and institutional affiliations.

\section{References}

1. Whyte RK, Jefferies AL. Red blood cell transfusion in newborn infants. Paediatr child health. 2014;19:213-22. (4)Epub 2014/05/ 24. PubMed PMID: 24855419; PubMed Central PMCID: PMCPMC4028649.

2. Ohls RK. Transfusions in the preterm infant. NeoReviews. 2007;8:e377-86.

3. Rajasekaran S, Kort E, Hackbarth R, Davis AT, Sanfilippo D, Fitzgerald R, et al. Red cell transfusions as an independent risk for mortality in critically ill children. J Intensive Care. 2016;4:2. Epub 2016/01/09. https://doi.org/10.1186/s40560-015-0122-3. PubMed PMID: 26744626; PubMed Central PMCID: PMCPMC4704419.

4. Hebert PC, Wells G, Blajchman MA, Marshall J, Martin C, Pagliarello G, et al. A multicenter, randomized, controlled clinical trial of transfusion requirements in critical care. Transfusion Requirements in Critical Care Investigators, Canadian Critical Care Trials Group. N Engl J Med. 1999;340:409-17. (6)Epub 1999/02/11. https://doi.org/10.1056/nejm199902113400601. PubMed PMID: 9971864

5. Salpeter SR, Buckley JS, Chatterjee S. Impact of more restrictive blood transfusion strategies on clinical outcomes: a meta-analysis and systematic review. Am J Med. 2014;127:124-31.e3. (2)Epub 2013/12/18. https://doi.org/10.1016/j.amjmed.2013.09.017. PubMed PMID: 24331453

6. Heeger LE, Counsilman CE, Bekker V, Bergman KA, Zwaginga $\mathrm{JJ}, \mathrm{Te}$ Pas $\mathrm{AB}$, et al. Restrictive guideline for red blood cell transfusions in preterm neonates: effect of a protocol change. Vox 
Sang. 2019;114:57-62. (1)Epub 2018/11/09. https://doi.org/10. 1111/vox.12724. PubMed PMID: 30407636.

7. Girelli G, Antoncecchi S, Casadei AM, Del Vecchio A, Isernia P, Motta M, et al. Recommendations for transfusion therapy in neonatology. Blood Transfus. 2015;13:484-97. (3)Epub 2015/10/ 09. https://doi.org/10.2450/2015.0113-15. PubMed PMID: 26445308 ; PubMed Central PMCID: PMCPMC4607607.

8. Bell EF, Strauss RG, Widness JA, Mahoney LT, Mock DM, Seward VJ, et al. Randomized trial of liberal versus restrictive guidelines for red blood cell transfusion in preterm infants. Pediatrics. 2005;115:1685-91. (6)Epub 2005/06/03. https://doi. org/10.1542/peds.2004-1884. PubMed PMID: 15930233; PubMed Central PMCID: PMCPMC2866196.

9. Demirel G, Celik IH, Aksoy HT, Erdeve O, Oguz SS, Uras N, et al. Transfusion-associated necrotising enterocolitis in very low birth weight premature infants. Transfus Med. 2012;22:332-7. (5) Epub 2012/06/29. https://doi.org/10.1111/j.1365-3148.2012. 01170.x. PubMed PMID: 22738152.

10. Faraday C, Hamad S, Jones K, Sim K, Cherian S, James A, et al. Characteristics and incidence of transfusion-associated necrotizing enterocolitis in the UKCharacteristics and incidence of transfusion-associated necrotizing enterocolitis in the UK. J Matern Fetal Neonatal Med. 2018 Epub 2018/06/28. https://doi. org/10.1080/14767058.2018.1494147. PubMed PMID: 29945481.

11. Kirpalani H, Whyte RK, Andersen C, Asztalos EV, Heddle N, Blajchman MA, et al. The Premature Infants in Need of Transfusion (PINT) study: a randomized, controlled trial of a restrictive (low) versus liberal (high) transfusion threshold for extremely low birth weight infants. J Pedia. 2006;149:301-7. (3)Epub 2006/08/ 31. https://doi.org/10.1016/j.jpeds.2006.05.011. PubMed PMID: 16939737

12. Lust C, Vesoulis Z, Jackups R Jr., Liao S, Rao R, Mathur AM. Early red cell transfusion is associated with development of severe retinopathy of prematurity. J Perinatol. 2019;39:393-400. (3). Epub 2018/11/22. https://doi.org/10.1038/s41372-018-0274-9. PubMed PMID: 30459388; PubMed Central PMCID: PMCPMC6391181.

13. Rashid N, Al-Sufayan F, Seshia MM, Baier RJ. Post transfusion lung injury in the neonatal population. J Perinatol. 2013;33:292-6. (4)Epub 2012/09/08. https://doi.org/10.1038/jp.2012.114. PubMed PMID: 22955289.

14. Widness JA, Seward VJ, Kromer IJ, Burmeister LF, Bell EF, Strauss RG. Changing patterns of red blood cell transfusion in very low birth weight infants. J Pedia. 1996;129:680-7. (5)Epub 1996/11/01. PubMed PMID: 8917234.

15. Maier RF, Obladen M, Muller-Hansen I, Kattner E, Merz U, Arlettaz R, et al. Early treatment with erythropoietin beta ameliorates anemia and reduces transfusion requirements in infants with birth weights below 1000 g. J Pedia. 2002;141:8-15. (1)Epub 2002/07/ 02. https://doi.org/10.1067/mpd.2002.124309. PubMed PMID: 12091844

16. Bell EF. Transfusion thresholds for preterm infants: how low should we go? J Pedia. 2006;149:287-9. (3)Epub 2006/08/31. https://doi.org/10.1016/j.jpeds.2006.06.033. PubMed PMID: 16939732.

17. LS d. Intracranial hemorrhage and vascular lesions in the neonate. In: Martin RJ FA, Walsh MC, editors. Fanaroff and Martin's Neonatal-Perinatal Medicine, 10th edn. Philadelphia: Elsevier Saunders; 2015.

18. Ellefson AM, Locke RG, Zhao Y, Mackley AB, Paul DA. Increased monocytes and bands following a red blood cell transfusion. J Perinatol. 2016;36:57-60. (1)Epub 2015/11/06. https:// doi.org/10.1038/jp.2015.153. PubMed PMID: 26540250.
19. The 'Effects of Transfusion Thresholds on Neurocognitive Outcome of Extremely Low Birth-Weight Infants (ETTNO)' Study: Background, Aims, and Study Protocol. Neonatology 2012;101(4):301-5. Epub 2012/02/03. https://doi.org/10.1159/000335030. PubMed PMID: 22298226.

20. Kirpalani H. 2012. https://www.nichd.nih.gov/sites/default/files/a bout/Documents/TOP_Protocol.pdf.

21. Dammann O. Inflammation and retinopathy of prematurity. Acta Paediatrica. 2010;99(7):975-7. Epub 2010/04/24. https://doi.org/ 10.1111/j.1651-2227.2010.01836.x. PubMed PMID: 20412106; PubMed Central PMCID: PMCPMC2902705.

22. Carson JL, Stanworth SJ, Alexander JH, Roubinian N, Fergusson DA, Triulzi DJ, et al. Clinical trials evaluating red blood cell transfusion thresholds: an updated systematic review and with additional focus on patients with cardiovascular disease. Am Heart J. 2018;200:96-101. Epub 2018/06/15. https://doi.org/10.1016/j. ahj.2018.04.007. PubMed PMID: 29898855.

23. Patel RM, Kandefer S, Walsh MC, Bell EF, Carlo WA, Laptook $\mathrm{AR}$, et al. Causes and timing of death in extremely premature infants from 2000 through 2011. N Engl J Med. 2015;372:331-40. (4)Epub 2015/01/22. https://doi.org/10.1056/ NEJMoa1403489. PubMed PMID: 25607427; PubMed Central PMCID: PMCPMC4349362.

24. Gregory KE, Deforge CE, Natale KM, Phillips M, Van Marter LJ. Necrotizing enterocolitis in the premature infant: neonatal nursing assessment, disease pathogenesis, and clinical presentation. Adv Neonatal Care. 2011;11:155-64. (3)quiz 65-6. Epub 2011/07/07. https://doi.org/10.1097/ANC.0b013e31821baaf4. PubMed PMID: 21730907; PubMed Central PMCID: PMCPMC3759524.

25. Patel RM, Knezevic A, Shenvi N, Hinkes M, Keene S, Roback JD, et al. Association of red blood cell transfusion, anemia, and necrotizing enterocolitis in very low-birth-weight infants. JAMA. 2016;315:889-97. (9)Epub 2016/03/05. https://doi.org/10.1001/ja ma.2016.1204. PubMed PMID: 26934258; PubMed Central PMCID: PMCPMC4805423.

26. Gephart SM. Transfusion-associated necrotizing enterocolitis: evidence and uncertainty. Adv Neonatal Care. 2012;12:232-6. (4)Epub 2012/ 08/07. https://doi.org/10.1097/ANC.0b013e31825e20ee. PubMed PMID: 22864004; PubMed Central PMCID: PMCPMC3414263.

27. Hay S, Zupancic JA, Flannery DD, Kirpalani H, Dukhovny D. Should we believe in transfusion-associated enterocolitis? Applying a GRADE to the literature. Semin Perinatol. 2017;41:80-91. (1)Epub 2016/11/22. https://doi.org/10.1053/j. semperi.2016.09.021. PubMed PMID: 27866662.

28. Maheshwari A, Patel RM, Christensen RD. Anemia, red blood cell transfusions, and necrotizing enterocolitis. Semin Pedia Surg. 2018;27:47-51. (1)Epub 2017/12/26. https://doi.org/10.1053/j. sempedsurg.2017.11.009. PubMed PMID: 29275817; PubMed Central PMCID: PMCPMC5776697.

29. Keir AK, McPhee AJ, Andersen CC, Stark MJ. Plasma cytokines and markers of endothelial activation increase after packed red blood cell transfusion in the preterm infant. Pedia Res. 2013;73:75-9. (1)Epub 2012/10/26. https://doi.org/10.1038/pr. 2012.144. PubMed PMID: 23095979.

30. Kenton AB, Hegemier S, Smith EO, O'Donovan DJ, Brandt ML, Cass DL, et al. Platelet transfusions in infants with necrotizing enterocolitis do not lower mortality but may increase morbidity. J Perinatol. 2005;25:173-7. (3)Epub 2004/12/04. https://doi.org/10. 1038/sj.jp.7211237. PubMed PMID: 15578029.

31. Curley A, Stanworth SJ, Willoughby K, Fustolo-Gunnink SF, Venkatesh V, Hudson C, et al. Randomized trial of platelettransfusion thresholds in neonates. N Engl J Med. 2018. Epub 2018/11/06. https://doi.org/10.1056/NEJMoa1807320. PubMed PMID: 30387697. 